\title{
Clinical challenges in the management of vaginal prolapse
}

This article was published in the following Dove Press journal:

International Journal of Women's Health

16 January 2014

Number of times this article has been viewed

\author{
Nazema Y Siddiqui \\ Autumn L Edenfield \\ Division of Urogynecology and \\ Reconstructive Surgery, Duke \\ University Medical Center, \\ Durham, NC, USA
}

Correspondence: Nazema Y Siddiqui

Division of Urogynecology and

Reconstructive Surgery, Department

of Obstetrics and Gynecology,

Duke University Medical Center 3192,

Durham, NC 277I0, USA

Tel +19194011006

Email nazema.siddiqui@duke.edu
Abstract: Pelvic organ prolapse is highly prevalent, and negatively affects a woman's quality of life. Women with bothersome prolapse may be offered pessary management or may choose to undergo corrective surgery. In choosing the most appropriate surgical procedure, there are many factors to consider. These may include the location(s) of anatomic defects, the severity of prolapse symptoms, the activity level of the woman, and concerns regarding the durability of the repair. In many instances, women and their surgeons are challenged to weigh the risks and benefits of native tissue versus mesh-augmented repairs. Though mesh-augmented repairs may offer better durability, they are also associated with unique complications, such as mesh erosion. Furthermore, newer surgical techniques of mesh placement via abdominal or vaginal routes may result in different outcomes compared to traditional techniques. Biologic grafts may also be considered to improve durability of a surgical repair, while avoiding potential complications of synthetic mesh. In this article, we review many of the clinical challenges that gynecologic surgeons face in the surgical management of vaginal prolapse. Furthermore, we review data that can help guide decision making when treating women with pelvic organ prolapse.

Keywords: pelvic organ prolapse, vaginal prolapse, surgery, sacrocolpopexy, sacrospinous ligament fixation, transvaginal mesh, uterosacral ligament suspension

\section{Introduction}

Pelvic organ prolapse (POP) is one of many pelvic floor disorders in women. Prolapse is a protrusion of the vaginal walls and/or uterus, resulting from descent of the pelvic organs. In general, "vaginal prolapse" includes multiple categories of pelvic support problems, such as uterine prolapse, posthysterectomy vaginal vault prolapse (enterocele), anterior vaginal wall prolapse (cystocele), and posterior vaginal wall prolapse (rectocele). These various support defects can occur in isolation or in combination with one another.

POP is highly prevalent, and can negatively impact a woman's quality of life. ${ }^{1-3}$ The etiology is multifactorial, and includes known risk factors of pregnancy and childbirth, increasing age, obesity, hysterectomy, connective tissue abnormalities, and conditions associated with increased abdominal strain. ${ }^{4}$ Prevalence estimates vary, since many women with mild prolapse are asymptomatic. Among women aged 50-79 years in the Women's Health Initiative, $41 \%$ had POP, with cystocele being the most common. ${ }^{5}$ Among 1,000 women seeking routine gynecologic care at six medical centers, 35\% had stage 2 prolapse (vaginal walls or uterus lie within $1 \mathrm{~cm}$ of hymen) and $2 \%$ had stage 3 prolapse (vaginal walls or uterus at least $1 \mathrm{~cm}$ beyond the hymen). ${ }^{6}$ 
Nearly $25 \%$ of women in the US suffer from pelvic floor disorders, and in the US the lifetime risk of undergoing a surgical procedure for prolapse or urinary incontinence is $11 \%$ by 80 years of age. ${ }^{1,7}$ In the US alone, approximately 200,000 women undergo surgery annually for prolapse, and approximately $29 \%$ require reoperation for recurrent prolapse. ${ }^{8}$ Recent projections estimate that the number of women undergoing surgery for POP will increase to approximately 250,000 by $2050 .{ }^{9}$ Thus, the estimated direct costs of prolapse surgery are over $\$ 1$ billion per year and will likely increase. ${ }^{8,10,11}$

Women with prolapse often complain of a sensation of a vaginal protrusion, seeing or feeling a bulge, or pelvic pressure or heaviness. ${ }^{4}$ In addition to bulge symptoms, women with prolapse can have associated symptoms of urinary incontinence, voiding dysfunction, defecatory dysfunction (eg, constipation or fecal incontinence), or sexual dysfunction..$^{1,4,12}$ These associated symptoms could result from prolapse or from other concomitant pelvic floor disorders, as bulge symptoms are the only specific symptoms of prolapse. ${ }^{4}$

Despite potential bother and associated symptoms, not all women with prolapse are symptomatic. While there is no obvious anatomic threshold that correlates with bothersome symptoms, the hymenal remnant seems to be an important landmark, as prolapse symptoms increase with descent to or beyond this level..$^{13-15}$ Women who are asymptomatic from their prolapse do not require treatment, and can continue under observation. However, women who are symptomatic have multiple treatment options, which include expectant management, the use of a pessary device, or pelvic surgery. For women who choose pessaries, these require ongoing maintenance and monitoring. Women who are younger, healthier, and sexually active tend to choose surgery over ongoing pessary use. ${ }^{16}$ Furthermore, women with a vaginal length less than $7 \mathrm{~cm}$, or those with a wider introitus (greater than $4 \mathrm{~cm}$ ) are less likely to be able to retain a pessary, ${ }^{17}$ and thus surgery may be the only treatment option in these instances. Ultimately, decisions about treatment tend to be based on the magnitude of symptoms and impact on a woman's quality of life. ${ }^{3}$ Thus, for women who choose surgery, we are often challenged to not only restore anatomy but also to find treatment choices that result in positive impact on both symptom burden and quality of life.

\section{Selecting a surgical procedure}

For women with prolapse, one of the biggest clinical challenges is selecting which surgery to perform. In addition to the route of surgery, the woman and her surgeon must decide on whether a native-tissue or graft-augmented repair is preferable. If a graft-augmented repair is chosen, there may be choices of different biologic grafts and synthetic mesh materials. Since there are risks and benefits to any approach, surgical counseling requires comprehensive knowledge of types of procedures, as well as the rates of prolapse recurrence and adverse events for each procedure (Table 1).

The decision for surgery for prolapse begins with a thorough examination. Prolapse severity is routinely measured with a quantitative pelvic exam, measuring the descent of the individual vaginal walls in the anterior, posterior, and apical compartments. The Baden-Walker halfway system ${ }^{18}$ and the POP quantitative system (POP-Q) ${ }^{19}$ are two common methods of assessing anatomic prolapse. The POP-Q examination is commonly used in research studies due to high reliability and standard classification of prolapse..$^{20-22}$ A standard quantitative exam is important, because it allows the surgeon to individually assess each wall of the vagina for prolapse, and in particular allows the surgeon to assess for the degree of apical prolapse. Women may have isolated anterior wall prolapse (cystocele), isolated posterior wall prolapse (rectocele), or may have a combination that also includes apical compartment prolapse (uterine prolapse or enterocele). If an apical defect is noted at the time of initial examination, it is important to include correction of the apex at the time of surgery, as this is highly associated with durability of the surgical repair. ${ }^{23}$

Surgical options may include isolated anterior or posterior vaginal wall repair if these are the only notable defects in support. For women who also have apical prolapse and are not sexually active, surgical options may include obliterative procedures, such as colpectomy/colpocleisis or LeFort colpocleisis. ${ }^{24}$ These procedures effectively close off the internal vaginal length and thus preclude future vaginal intercourse, but are also very durable repairs. ${ }^{25}$ For women with a component of apical prolapse who are sexually active, reconstructive procedures should be offered, as these procedures provide support while restoring the vaginal contour. Reconstructive surgery for apical prolapse can be performed via vaginal or abdominal routes, and also can be performed entirely using sutures and native tissues or can be augmented with either a synthetic mesh or biologic graft. Choosing between treatment options, particularly surgical options, involves weighing risks and benefits of each type of intervention. In addition to the anatomic location and severity of the prolapse, factors such as the overall health and activity 


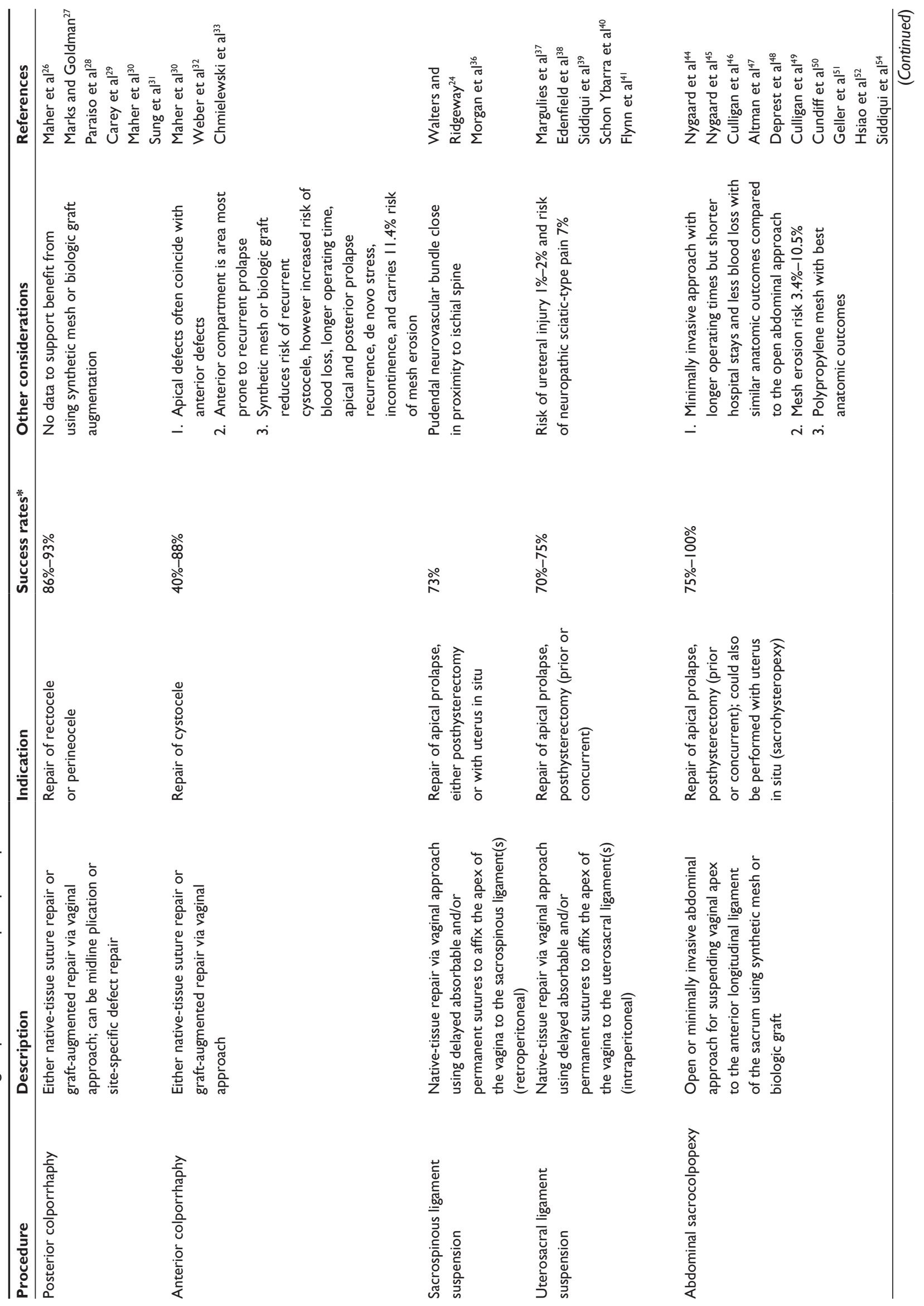


level of the patient and other concurrent pelvic floor symptoms all contribute to the decision of treatment choice.

\section{Isolated posterior compartment defects}

If a woman has an isolated rectocele without any apical prolapse, surgical decision making can be fairly easy. Traditional posterior colporrhaphy uses stitches and the woman's own tissue to reestablish support in the posterior vagina. Recurrence occurs in 7\%-14\% of women after 1 year. ${ }^{26-28}$ Randomized trials comparing traditional posterior colporrhaphy to biologic graft- or synthetic mesh-augmented repairs have shown equivalent or superior anatomic outcomes with traditional posterior colporrhaphy, without the complications associated with grafts. ${ }^{28,29}$ Thus, at this time there are no data to support using mesh or graft augmentation for an isolated posterior compartment defect..$^{30,31}$

\section{Isolated anterior compartment defects}

In women with a presumed isolated cystocele, it is essential to assess thoroughly for an apical compartment defect. This is important, because anterior and apical prolapse are highly correlated and often coexist. ${ }^{23}$ If there are co-occurring anterior and apical defects, failure to address the vaginal apex may result in early recurrence of prolapse, regardless of which surgery is performed. If the surgeon concludes that the apex of the vagina is well supported and there is in isolated anterior compartment defect, there are still some difficult choices regarding surgical repair.

The anterior vaginal compartment is the area most prone to prolapse recurrence, with $28 \%-40 \%$ of women experiencing prolapse recurrence after traditional anterior colporrhaphy. ${ }^{30,32}$ For this compartment in particular, the way that recurrent prolapse is defined seems to be very important. Historically, we focused more on anatomic outcomes and examination findings. However, recent data show that a woman's symptoms of vaginal bulge correlate best with success. ${ }^{14}$ Thus, a woman can have a recurrent minor cystocele that the surgeon deems as a "recurrence," but she may be completely asymptomatic with regard to bulge symptoms and perceive her surgery as a "success." A striking example of this is demonstrated by an analysis of a randomized trial of three techniques of anterior colporrhaphy. ${ }^{32}$ The initial publication of this study defined surgical success using strict anatomic measurements, and with this definition only $40 \%$ of subjects had a "successful" surgery. The authors later reanalyzed the same data using a clinically relevant definition 
of surgical success, which included 1) no prolapse beyond the hymen, and 2) the absence of prolapse symptoms. ${ }^{33} \mathrm{With}$ this new composite definition, $88 \%$ of women met the definition of success, which correlates with the occurrence of only one of 114 (1\%) of these subjects undergoing repeat surgery for prolapse. Thus, when assessing outcomes after prolapse surgery, anatomy and symptoms are both important, and both should be incorporated into the definition of surgical success. That being said, in the anterior compartment there is evidence suggesting that a biologic graft or synthetic mesh may allow for more durability of a surgical repair. ${ }^{31}$

In a recent large systematic review, synthetic mesh or biologic grafts during anterior colporrhaphy were shown to reduce the risk of recurrent cystocele over traditional suture reinforcement alone. ${ }^{30}$ This was evident both on clinical examination and in the reduction of prolapse symptoms. Native-tissue anterior colporrhaphy is associated with more recurrent anterior prolapse compared to absorbable polyglactin mesh (relative risk [RR] 1.39, 95\% confidence interval [CI] 1.02-1.90) or porcine dermis (RR 2.08, 95\% CI 1.08-4.01); however, there were no differences in subjective awareness of prolapse. ${ }^{30}$ Native-tissue repairs compared to colporrhaphy with polypropylene (permanent) mesh have an increased risk of recurrent anterior prolapse (RR 3.15, 95\% CI 2.50-3.96) and an increased risk of awareness of prolapse (RR 1.57, 95\% CI 1.18-2.07). ${ }^{30}$ However, in the native-tissue and mesh groups, there were similar rates of reoperation for prolapse and no significant differences in quality of life. ${ }^{30}$ Mesh erosion (exposure or extrusion into the vagina) occurs in $11.4 \%$ after mesh-augmented anterior vaginal wall repair. ${ }^{30}$ Furthermore, mesh repairs are associated with increased blood loss, longer operating time, apical and posterior prolapse recurrences, and de novo stress incontinence. ${ }^{30}$ Thus, there are data suggesting that mesh augmentation results in improved anatomic support for anterior wall prolapse, but for women who are choosing surgery to improve quality of life, the potential complications may outweigh the benefits of improved support and durability. For surgeons who are considering a mesh-augmented repair for cystocele, it is important to discuss durability and potential complications, so that the woman can choose the best repair, based on her priorities.

\section{Apical compartment defects}

During the preoperative evaluation, if a surgeon identifies an apical compartment defect, either alone or in combination with other support defects, surgical decision making becomes even more complex. In this situation, it is important to again highlight that for non-sexually active women, obliterative surgeries may offer the best durability, ${ }^{25}$ and are associated with very high postoperative satisfaction and quality of life. ${ }^{34,35}$ However, for the numerous women who wish to remain sexually active, reconstructive surgeries should be offered. These surgeries may involve nativetissue repairs, biologic graft-augmented repairs, or synthetic mesh-augmented repairs, and could be performed from an abdominal or vaginal approach. Thus, there are many options to consider. We will first discuss the traditional surgical procedures for apical prolapse, including native-tissue repairs and abdominal sacrocolpopexy. Later in this review, we will discuss other less invasive mesh-augmented repairs, such as minimally invasive sacrocolpopexy and transvaginal mesh.

\section{Native-tissue apical repairs}

Though many variations of apical native-tissue prolapse repairs exist, two very common procedures are the sacrospinous ligament fixation (Figure 1) and the uterosacral ligament suspension (Figure 2). These procedures involve using delayed absorbable and/or permanent sutures to affix the apex of the vagina to pelvic ligaments. Sacrospinous ligament fixation (SSLF) anchors the vaginal apex to the sacrospinous ligament, and does not require intraperitoneal entry. Thus, it can be performed after hysterectomy or while leaving the uterus in situ. Although it can be performed bilaterally, the stitches are often placed unilaterally on one side or the other. ${ }^{24}$ This results in the vaginal apex being slightly deviated to the

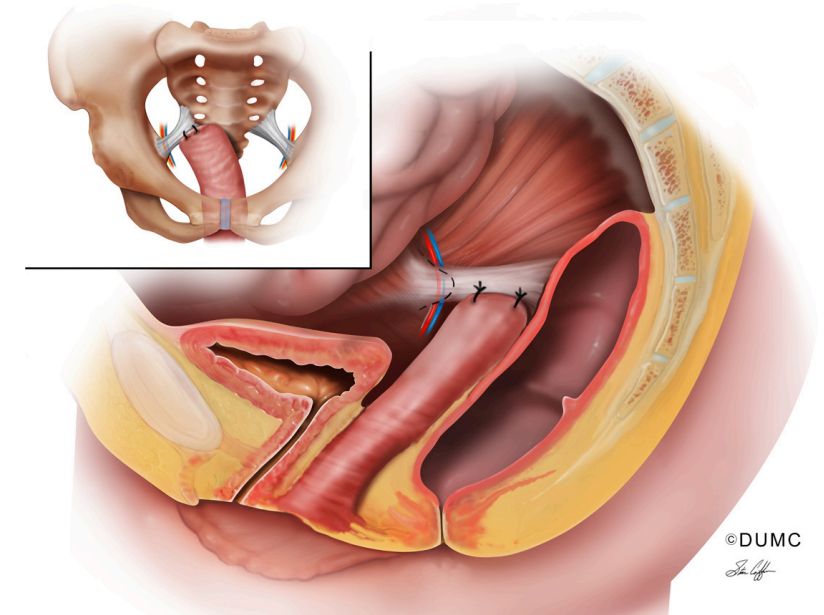

Figure I Sacrospinous ligament fixation. The apex of the vagina is affixed with two sutures to the right sacrospinous ligament. The ischial spine is depicted with the pudendal neurovascular bundle (nerve, artery, vein) in close proximity to the ischial spine. This procedure may be performed unilaterally or bilaterally (attaching to both sacrospinous ligaments) with one or more permanent or delayed absorbable sutures. 


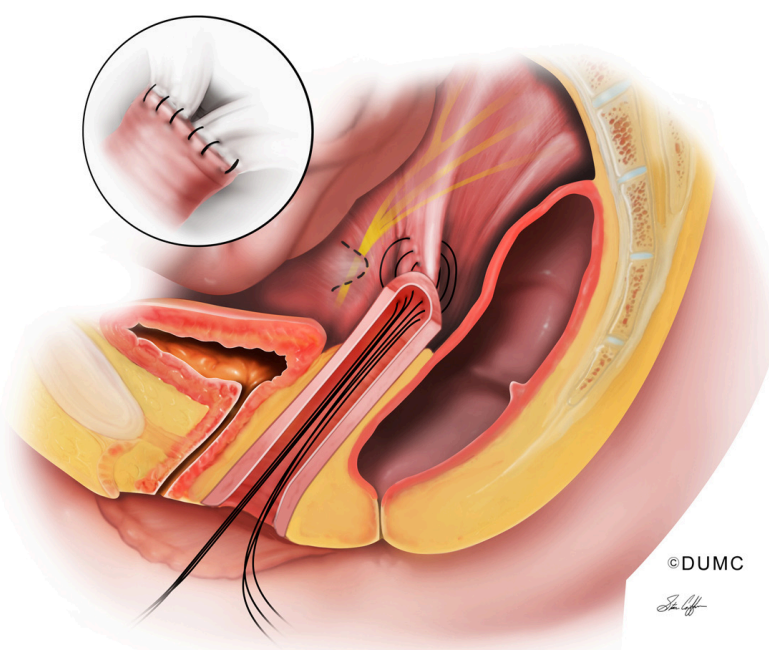

Figure 2 Uterosacral ligament suspension. Stitches are placed through an open vaginal cuff into the uterosacral ligament. Image depicts three stitches in the right uterosacral ligament, which are then brought through the vaginal cuff (if permanent stitches are used, they are placed through a partial-thickness segment of the vaginal cuff and are not brought into the vaginal cavity). The image also shows the course of the sacral nerve roots, which exit various sacral foramina and join to form the lumbosacral trunk. These nerves lie underneath the peritoneum, deep in the pelvis. Inset depicts view from inside the abdomen after stitches are tied down, where the vaginal cuff is affixed to bilateral uterosacral ligaments.

right or left, in addition to the downward deviation that is required for the apex to reach to the sacrospinous ligament. When stitches are placed into the sacrospinous ligament, care should be taken to avoid the pudendal neurovascular bundle, which lies in close proximity to the ischial spine. This is a potential source of complications, such as hemorrhage and nerve injury (pudendal neuropathy). Recurrence of prolapse after SSLF varies based on the outcome definitions that are used, but occurs in up to $27 \%$ of women. ${ }^{36}$

Uterosacral ligament suspension (USLS) is performed in a transperitoneal fashion, and is often performed vaginally after a hysterectomy has already been performed. USLS can also be accomplished laparoscopically, but data are lacking regarding long-term outcomes after the laparoscopic approach. For vaginal USLS, prolapse may recur in $25 \%-30 \%$ of women. ${ }^{37,38}$ Though ureteral injury is thought to be the most likely complication, risk of ureteral injury is actually much lower than initially reported, at $1 \%-2 \%$ in recent large series. ${ }^{37,38}$ Sciatic-type pain can also occur as a result of compression or entrapment of sacral nerve roots with USLS stitches, ${ }^{39,40}$ and this may happen in up to $7 \%$ of women. ${ }^{41,42}$ On the other hand, the benefit of USLS is that it restores the vagina to its usual axis without involving a permanent mesh implant (Figure 2).

As noted, both SSLF and USLS have a similar $\sim 25 \%$ risk of recurrent prolapse. To date, there has been only one randomized controlled trial comparing these two native-tissue repairs (SSLF and USLS), and data regarding long-term prolapse recurrence are still being collected. ${ }^{43}$ Thus, we do not yet know which of these two procedures may be superior, and often the decision on whether to perform SSLF or USLS is based on surgeon preference.

\section{Abdominal sacrocolpopexy}

Both SSLF and USLS have low risks of potential complications, but are limited in their relatively high rates of prolapse recurrence. Thus, surgeons have looked to other options that may afford more durability when addressing apical prolapse. Abdominal sacrocolpopexy (ASC) is a surgical technique for correction of apical and/or advanced anterior wall prolapse that is performed with graft augmentation (Figure 3). Options for graft augmentation include synthetic mesh materials, such as permanent mesh (woven polyester, polypropylene, or expanded polytetrafluoroethylene, [ePTFE]). In addition, there are biologic materials, including xenografts (porcine dermis or bovine tissues), and allografts such as cadaveric fascia. Estimates for prolapse recurrence after ASC range from $0 \%$ to $22 \%$. ${ }^{44}$ This wide variation could be attributed to variability in graft materials. However, even when using synthetic mesh, recent data have challenged the durability of the ASC procedure, showing approximately $25 \%$ anatomic prolapse recurrence when patients were evaluated after 7 years. ${ }^{45}$ Notably, this follow-up period is significantly longer than most reported studies.

Regardless, when compared to native-tissue vaginal vault repairs, ASC has demonstrated more durability in multiple randomized trials. ${ }^{30}$ The benefits in durability of

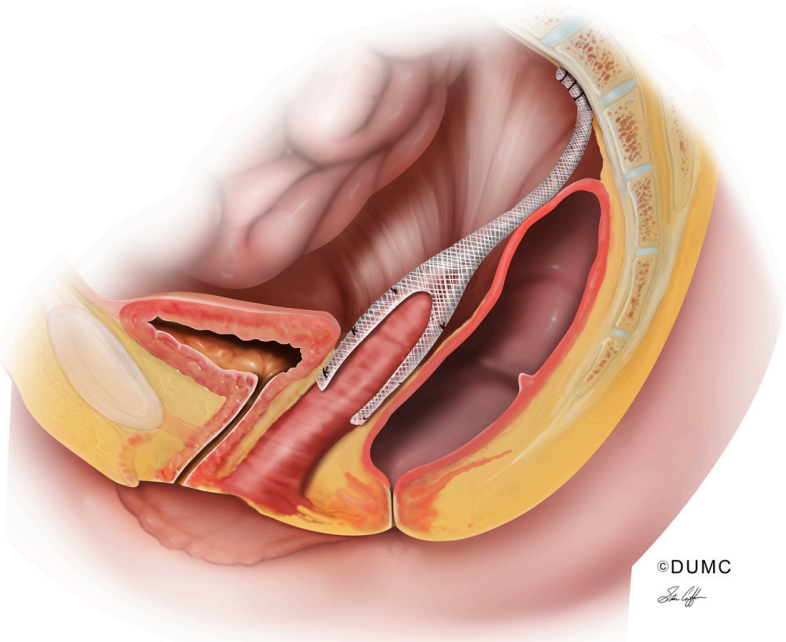

Figure 3 Mesh sacrocolpopexy. Whether performed abdominally or via minimally invasive techniques, mesh is attached to the anterior and/or posterior walls of the vagina. The "tail" of the mesh is attached to the anterior longitudinal ligament of the sacrum. 
ASC must also be weighed against longer operating times, longer recovery, and potential mesh complications. The use of permanent mesh has certainly come under debate, and other materials, such as biologic grafts and cadaveric tissue, have been explored. Some authors have shown similar outcomes when sacrocolpopexy is performed with xenograft compared to synthetic mesh, ${ }^{46,47}$ but a large randomized trial with medium-term follow-up (mean 33 months) showed that xenograft was associated with more apical failures and reoperations for prolapse. ${ }^{48}$ Another study of 100 women randomized to either cadaveric fascia or polypropylene mesh during sacrocolpopexy found that recurrent prolapse was worse 1 year postoperatively in the fascia group (32\%) compared to the synthetic mesh group (9\%). ${ }^{49}$

One of the major concerns with synthetic mesh is mesh erosion. Estimates for the risk of mesh erosion after ASC range from $3.4 \%$ to $10.5 \% .^{44,50}$ In a multicenter surgical trial of 322 women who underwent ASC with a variety of surgical techniques and selected graft materials, the rate of mesh/suture erosion was $6 \%$ at 2 years after surgery and $10.5 \%$ at 7 years after surgery. ${ }^{45,50}$ Most of the procedures were performed with synthetic mesh (92\%) with $42 \%$ woven polyester, $48 \%$ polypropylene, and $6 \%$ ePTFE, while synthetic absorbable grafts were not used in this study. ${ }^{50}$ The use of ePTFE was associated with an increased risk of erosion, as four of the first five reports of mesh or suture erosions involved Gore-Tex mesh, so the use of this material was discontinued for the remainder of the study..$^{50}$ Generally, polypropylene mesh has become the favored synthetic material for clinical use.

Since prolapse surgery is offered to improve quality of life, when offering ASC it is important to consider that it may be a more durable approach, but may also require repeat surgery for mesh issues in up to $5 \%-10 \%$ of patients. This is in addition to the $5 \%-10 \%$ of patients who require pessary or surgery for a recurrent bulge. Many women consider repeat treatment for prolapse and repeat treatment for mesh complications to have similar impingement on their quality of life. Thus, when considering whether or not to perform a native-tissue repair or a mesh-augmented repair like ASC, it may be more accurate to weigh the risk of repeat treatment for any reason when counseling the patient.

\section{Minimally invasive sacrocolpopexy}

Though ASC is more durable than native-tissue vaginal repair, open abdominal surgery is also more morbid. As noted in a Cochrane review, the benefits in durability of ASC must be weighed against longer operating times and a longer recovery period. ${ }^{30}$ Thus, in the last decade there have been concerted efforts to provide the durability of a mesh-augmented repair in a less invasive manner. In many centers, ASC has been transitioned to a minimally invasive approach. Laparoscopy, both with and without robotic assistance, has been used to provide mesh sacrocolpopexy without an open abdominal incision. Though operating times are still longer than vaginal surgery, multiple studies of minimally invasive surgery, including laparoscopic sacrocolpopexy (LSC) and robotic sacrocolpopexy (RSC), show shorter hospital stays and less blood loss compared to the open abdominal approach..$^{51,52}$ In addition, there is presumed quicker recovery and less pain, and all of these factors are important considerations for women who are choosing to undergo elective surgery.

Though short-term outcomes such as recovery time may be better with minimally invasive sacrocolpopexy, it is important to also consider whether prolapse outcomes are similar. In other words, are we performing an equivalent surgery when we move from the open to the laparoscopic approach? In a group of Medicare beneficiaries in the US, laparoscopic (including robotic) sacrocolpopexy was associated with an increased rate of reoperation for anterior vaginal wall prolapse (3.4\% versus $1 \%$ in ASC). ${ }^{53}$ Presumably, these anterior wall recurrences could occur with LS surgery because the mesh may not be placed as low on the anterior vaginal wall. Notably this database only included 176 LS procedures from 2004 to 2008, and it is uncertain how such factors as the learning curve for LS surgery might affect these data. Multiple other comparative studies have shown similar anatomic outcomes 1 year after minimally invasive and traditional open abdominal sacrocolpopexy. ${ }^{52,54}$ This includes a randomized trial in 53 subjects undergoing LSC versus RSC in the $\mathrm{UK}^{55}$ and a cohort study comparing 125 RSC to 322 ASC procedures in the US. ${ }^{54}$

Even for those who adopt minimally invasive sacrocolpopexy, there is an ongoing debate about whether traditional laparoscopy or robotic-assisted laparoscopy should be used for this procedure. One randomized trial of LSC versus RSC showed no difference in anatomic prolapse or bulge symptoms 1 year after surgery, demonstrating that long-term outcomes after these two minimally invasive approaches may be similar. ${ }^{28}$ However, in this study, the authors showed that robotic-assisted laparoscopy was significantly more costly, mainly because of a longer duration of surgery (265 minutes for RSC versus 199 minutes for LSC). With more experience, particularly after the first 20 cases, robotic sacrocolpopexies are now being performed in 101-192 minutes. ${ }^{56,57}$ Thus, for 
high-volume robotic surgeons who have shorter operating times, it appears that robotic sacrocolpopexy may be less costly than originally thought.

\section{Transvaginal mesh}

Another way in which surgeons have tried to provide the benefits of a mesh-augmented repair with a less invasive approach is by offering transvaginal mesh repairs (Figure 4). A recent study of a large US health care claims database shows that from 2005 to 2010 the rate of all procedures for prolapse using mesh grafts increased, with vaginal mesh surgeries constituting the vast majority (approximately 75\%). ${ }^{58}$ These typically involved permanent meshes that were placed with a vaginal approach using trocars to guide arms of mesh into place. However, the landscape of available transvaginal mesh products is rapidly changing, particularly following the 2011 US Food and Drug Administration (FDA) safety communication, which expressed concern regarding adverse events and complications associated with transvaginal mesh prolapse repairs. ${ }^{59}$ In this communication, an FDA-sponsored systematic review found that erosion of mesh through the vagina was the most consistent and common mesh-related complication, and that mesh contraction (shrinkage) was a previously unidentified risk of transvaginal mesh repairs. . $^{59,60}$ These complications may lead to pelvic pain, dyspareunia, or the inability to achieve vaginal intercourse. Notably, the FDA did not find conclusive evidence that transvaginally placed mesh improves outcomes any more than traditional prolapse repair without mesh, and may expose patients to greater risk.

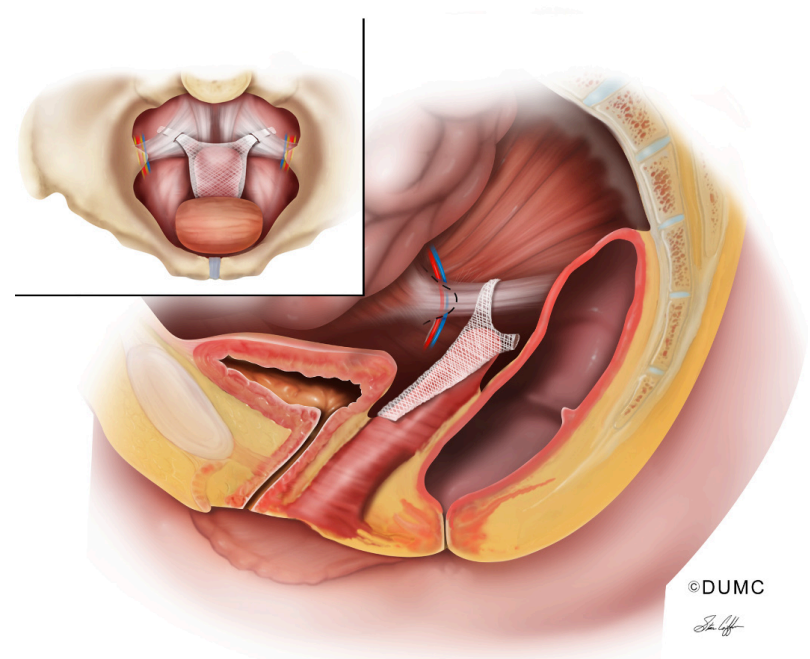

Figure 4 Transvaginal mesh. Image of an anterior/apical transvaginal mesh. The body of the mesh lies over the anterior vaginal wall and apex of the vagina. The "arms" of the mesh are placed into the sacrospinous ligaments bilaterally. The ischial spine is depicted with the pudendal neurovascular bundle (nerve, artery, vein) in close proximity to the ischial spine.
In a systematic review of randomized trials of transvaginal prolapse repair in multiple compartments (anterior, posterior, and apical), polypropylene mesh trocar-delivered "kits" have a mesh-exposure rate of $18 \%$, with half of those women undergoing reoperation for mesh complications. ${ }^{30}$ Another recent systematic review found that mesh-exposure rates following transvaginal prolapse repair vary widely across studies, with a range of 0 to $29.7 \% .{ }^{61}$ There are improved anatomical outcomes after transvaginal prolapse repair with permanent mesh augmentation; however, no differences are found in symptoms or quality of life. ${ }^{30}$ Ultimately, despite fewer reoperations for prolapse, total reoperations are highest for transvaginal mesh compared to other procedures, and these are mainly due to mesh complications. ${ }^{62}$

In the face of these findings, the US FDA has recommended that surgeons obtain specialized training for each mesh-placement technique and counsel patients thoroughly about potential mesh-related complications. In addition, the FDA recommends that health care providers choose mesh surgery only after weighing the risks and benefits against other alternatives. ${ }^{59}$ Surgeons and researchers recognize that mesh augmentation aids in durability of prolapse repair, but it is essential also to minimize complications. Therefore, researchers are keenly interested in investigating the optimal materials for mesh or graft augmentation that would maintain a long-lasting and durable prolapse repair, while minimizing risk of erosion through the vaginal tissue or surrounding organs.

Because of the existing challenges with durability and mesh-related complications for prolapse repair, we continue to seek better surgical options. Newer transvaginal mesh techniques continue to be developed where modifications in how mesh arms are placed into the sacrospinous ligament and where the location of vaginal incisions are placed may alter the number of complications. ${ }^{63}$ These newer techniques continue to be evaluated, and are the focus of a current US National Institutes of Health-sponsored randomized trial. ${ }^{64}$ Given that abdominal and minimally invasive sacrocolpopexy has inherent surgical risks of increased operating time, cost, and surgical risks associated with the intraabdominal technique, ${ }^{30,44}$ interest will continue in optimizing transvaginal prolapse-repair techniques with improved graft augmentation. However, with the recent controversy surrounding vaginal mesh, it is important to counsel patients regarding the potential risks and benefits, and in particular be able to offer nonmesh approaches for those patients who do not wish to be subject to the risks associated with permanent mesh. Societies are now publishing guidelines 
to help guide clinicians regarding the training needed to be able to offer prolapse surgeries and the counseling process that women should undergo before they opt for surgical correction of prolapse. ${ }^{65}$

Clinicians are often challenged to consider which is the most appropriate surgical procedure to perform for their patients. Often, these decisions rely on detailed discussions regarding risks and benefits, and there may be subtle nuances that make one procedure slightly more favorable than another for any given patient. A thorough knowledge of treatment options and the risks and benefits associated with each procedure is essential.

\section{Other considerations}

As noted earlier, in addition to bulge symptoms, women with prolapse can have associated symptoms of urinary incontinence, defecatory dysfunction, or sexual dysfunction. Since prolapse is often intertwined with other pelvic floor disorders, addressing a bulge alone may not improve a woman's quality of life to the degree that is desired. This is another challenge for surgeons who are generally wishing for successful quality-of-life outcomes for their patients. Thus, during a preoperative evaluation it is helpful to assess for other concomitant symptoms to develop a comprehensive treatment plan.

Prolapse and stress urinary incontinence (SUI) can occur concurrently, and women with prolapse who are continent have an increased risk of developing de novo SUI after surgical prolapse repair. ${ }^{66,67}$ Therefore, addressing SUI at the time of surgical intervention for prolapse is an important consideration for improving overall quality of life and decreasing pelvic floor symptoms. ${ }^{25}$ Performing an anti-incontinence procedure at the time of prolapse repair is effective in reducing the risk of occult SUI postoperatively. ${ }^{30}$ A multicenter randomized controlled trial of Burch retropubic urethropexy at the time of ASC showed significantly decreased risk of SUI if a Burch urethropexy was performed prophylactically. ${ }^{66}$ Three months postoperatively, 24\% of those who underwent Burch urethropexy met objective criteria for SUI compared to $44 \%$ of those who did not have a Burch procedure; only $6 \%$ reported bothersome SUI symptoms, while $25 \%$ of control subjects reported bothersome SUI symptoms. ${ }^{66}$

Despite the efficacy of Burch urethropexy at the time of prolapse repair, this is an abdominal procedure with associated risks and comorbidities. Many surgeons have implemented the use of midurethral mesh slings for the routine treatment of SUI, given that mesh-sling procedures are as effective as retropubic urethropexy and traditional pubovaginal sling procedures with fewer postoperative complications. ${ }^{68}$ A multicenter randomized trial of 337 continent women who underwent vaginal prolapse surgery and were randomized to either midurethral sling or no sling showed a significantly decreased risk of postoperative SUI if a prophylactic sling was performed. ${ }^{67}$ Three months postoperatively, SUI occurred in $24 \%$ of those who received a sling compared to $49 \%$ of those who did not. ${ }^{67}$ This difference persisted at 12 months, with $23 \%$ and $43 \%$ reporting SUI in the sling and no-sling groups, respectively. ${ }^{67}$

In addition to SUI, defecatory symptoms can often occur along with POP. Women may have symptomatic prolapse in anterior and/or apical compartments, but during their evaluation, defecatory dysfunction or a small rectocele may be identified. In these situations, surgeons are often challenged regarding whether or not to add surgical repair for a minimally symptomatic defect in the posterior compartment. In women with pelvic floor disorders, $36 \%$ meet the criteria for constipation, ${ }^{69}$ and compared to control subjects with normal pelvic support, women with prolapse have a fourfold increase in constipation. ${ }^{70}$ Obstructive defecation is a bowel symptom that is often associated with posterior compartment prolapse in particular, ${ }^{71}$ and pelvic reconstructive surgery with posterior repair has been shown to improve overall bowel function. ${ }^{72,73}$ In 99 women undergoing rectocele repair, 96 underwent concurrent prolapse repair in another compartment, and bowel symptoms significantly improved in all patients 1 year after surgery. ${ }^{73}$ Among 211 women who underwent abdominal sacrocolpopexy, bowel symptoms improved significantly among those who did and did not have a rectocele repair. ${ }^{72}$ Though apical prolapse surgery alone may help with bowel issues, adding a rectocele repair may offer further improvements in bowel function. Therefore, addressing bowel symptoms preoperatively and tailoring surgical repair and approach with a possible rectocele repair is an important consideration when planning for surgical reconstruction.

\section{Conclusion}

Treatment of POP is clinically challenging because of the need to address pelvic floor symptoms, provide a high quality of life, and minimize complications. There is a wide range of surgical options that may be used. The surgeon who treats prolapse should be able to discuss and offer native-tissue procedures for prolapse. In addition, for clinically challenging situations or women with recurrent prolapse, mesh-augmented repairs may be considered. A thorough knowledge of mesh and graft options, as well as knowledge of prolapse recurrence 
and adverse event rates, can help guide clinicians in counseling their patients effectively. Ultimately, this will allow surgeons to choose personalized treatment options that best align with a woman's lifestyle and treatment goals.

\section{Acknowledgment}

The authors wish to acknowledge Stan Coffman for assistance with medical illustrations.

\section{Disclosure}

Dr Edenfield has no potential conflicts of interest. Dr Siddiqui receives research grant funding from Medtronic, Inc, and has received reimbursement for travel to educational meetings from Intuitive Surgical, Inc.

\section{References}

1. Nygaard I, Barber MD, Burgio KL, et al. Prevalence of symptomatic pelvic floor disorders in US women. JAMA. 2008;300(11):1311-1316.

2. Nygaard I, Bradley C, Brandt D. Pelvic organ prolapse in older women: prevalence and risk factors. Obstet Gynecol. 2004;104(3):489-497.

3. Jelovsek JE, Barber MD. Women seeking treatment for advanced pelvic organ prolapse have decreased body image and quality of life. Am J Obstet Gynecol. 2006;194(5):1455-1461.

4. Jelovsek JE, Maher C, Barber MD. Pelvic organ prolapse. Lancet. 2007;369(9566):1027-1038.

5. Hendrix SL, Clark A, Nygaard I, Aragaki A, Barnabei V, McTiernan A. Pelvic organ prolapse in the Women's Health Initiative: gravity and gravidity. Am J Obstet Gynecol. Jun 2002;186(6):1160-1166.

6. Swift S, Woodman P, O'Boyle A, et al. Pelvic Organ Support Study (POSST): the distribution, clinical definition, and epidemiologic condition of pelvic organ support defects. Am J Obstet Gynecol. 2005;192(3):795-806.

7. Olsen AL, Smith VJ, Bergstrom JO, Colling JC, Clark AL. Epidemiology of surgically managed pelvic organ prolapse and urinary incontinence. Obstet Gynecol. 1997;89(4):501-506.

8. Boyles SH, Weber AM, Meyn L. Procedures for pelvic organ prolapse in the United States, 1979-1997. Am J Obstet Gynecol. 2003;188(1): $108-115$.

9. Wu JM, Kawasaki A, Hundley AF, Dieter AA, Myers ER, Sung VW. Predicting the number of women who will undergo incontinence and prolapse surgery, 2010 to 2050. Am J Obstet Gynecol. 2011;205(3):230. e1-e5.

10. Erekson EA, Lopes VV, Raker CA, Sung VW. Ambulatory procedures for female pelvic floor disorders in the United States. Am J Obstet Gynecol. 2010;203(5):497. e1-e5.

11. Subak LL, Waetjen LE, van den Eeden S, Thom DH, Vittinghoff E, Brown JS. Cost of pelvic organ prolapse surgery in the United States. Obstet Gynecol. 2001;98(4):646-651.

12. Ellerkmann RM, Cundiff GW, Melick CF, Nihira MA, Leffler K, Bent AE. Correlation of symptoms with location and severity of pelvic organ prolapse. Am J Obstet Gynecol. 2001;185(6):1332-1337; discussion 1337-1338.

13. Swift SE, Tate SB, Nicholas J. Correlation of symptoms with degree of pelvic organ support in a general population of women: what is pelvic organ prolapse? Am J Obstet Gynecol. 2003;189(2):372-377; discussion 377-379.

14. Barber MD, Brubaker L, Nygaard I, et al. Defining success after surgery for pelvic organ prolapse. Obstet Gynecol. 2009;114(3):600-609.

15. Tan JS, Lukacz ES, Menefee SA, Powell CR, Nager CW. Predictive value of prolapse symptoms: a large database study. Int Urogynecol J Pelvic Floor Dysfunct. 2005;16(3):203-209; discussion 209.
16. Clemons JL, Aguilar VC, Sokol ER, Jackson ND, Myers DL. Patient characteristics that are associated with continued pessary use versus surgery after 1 year. Am J Obstet Gynecol. 2004;191(1):159-164.

17. Clemons JL, Aguilar VC, Tillinghast TA, Jackson ND, Myers DL. Risk factors associated with an unsuccessful pessary fitting trial in women with pelvic organ prolapse. Am J Obstet Gynecol. 2004;190(2): 345-350.

18. Baden WF, Walker TA. Genesis of the vaginal profile: a correlated classification of vaginal relaxation. Clin Obstet Gynecol. 1972;15(4):1048-1054.

19. Bump RC, Mattiasson A, Bo K, et al. The standardization of terminology of female pelvic organ prolapse and pelvic floor dysfunction. $\mathrm{Am}$ J Obstet Gynecol. 1996;175(1):10-17.

20. Hall AF, Theofrastous JP, Cundiff GW, et al. Interobserver and intraobserver reliability of the proposed International Continence Society, Society of Gynecologic Surgeons, and American Urogynecologic Society pelvic organ prolapse classification system. Am J Obstet Gynecol. 1996;175(6):1467-1470; discussion 1470-1461.

21. Kobak WH, Rosenberger K, Walters MD. Interobserver variation in the assessment of pelvic organ prolapse. Int Urogynecol J Pelvic Floor Dysfunct. 1996;7(3):121-124.

22. Muir TW, Stepp KJ, Barber MD. Adoption of the pelvic organ prolapse quantification system in peer-reviewed literature. Am J Obstet Gynecol. 2003;189(6):1632-1635; discussion 1635-1636.

23. Rooney K, Kenton K, Mueller ER, FitzGerald MP, Brubaker L. Advanced anterior vaginal wall prolapse is highly correlated with apical prolapse. Am J Obstet Gynecol. 2006;195(6):1837-1840.

24. Walters MD, Ridgeway BM. Surgical treatment of vaginal apex prolapse. Obstet Gynecol. 2013;121(2 Pt 1):354-374.

25. Zebede S, Smith AL, Plowright LN, Hegde A, Aguilar VC, Davila GW. Obliterative LeFort colpocleisis in a large group of elderly women. Obstet Gynecol. 2013;121(2 Pt 1):279-284.

26. Maher CF, Qatawneh AM, Baessler K, Schluter PJ. Midline rectovaginal fascial plication for repair of rectocele and obstructed defecation. Obstet Gynecol. 2004;104(4):685-689.

27. Marks BK, Goldman HB. What is the gold standard for posterior vaginal wall prolapse repair: mesh or native tissue? Curr Urol Rep. 2012;13(3):216-221.

28. Paraiso MF, Barber MD, Muir TW, Walters MD. Rectocele repair: a randomized trial of three surgical techniques including graft augmentation. Am J Obstet Gynecol. 2006;195(6):1762-1771.

29. Carey M, Higgs P, Goh J, et al. Vaginal repair with mesh versus colporrhaphy for prolapse: a randomised controlled trial. BJOG. 2009;116(10):1380-1386.

30. Maher C, Feiner B, Baessler K, Schmid C. Surgical management of pelvic organ prolapse in women. Cochrane Database Syst Rev. 2013;4:CD004014.

31. Sung VW, Rogers RG, Schaffer JI, et al. Graft use in transvaginal pelvic organ prolapse repair: a systematic review. Obstet Gynecol. 2008;112(5):1131-1142.

32. Weber AM, Walters MD, Piedmonte MR, Ballard LA. Anterior colporrhaphy: a randomized trial of three surgical techniques. $\mathrm{Am} J$ Obstet Gynecol. 2001;185(6):1299-1304; discussion 1304-1296.

33. Chmielewski L, Walters MD, Weber AM, Barber MD. Reanalysis of a randomized trial of 3 techniques of anterior colporrhaphy using clinically relevant definitions of success. Am J Obstet Gynecol. 2011;205(1):69. e1-e8.

34. Crisp CC, Book NM, Smith AL, et al. Body image, regret, and satisfaction following colpocleisis. Am J Obstet Gynecol. Epub May 9, 2013.

35. Fitzgerald MP, Richter HE, Bradley CS, et al. Pelvic support, pelvic symptoms, and patient satisfaction after colpocleisis. Int Urogynecol J Pelvic Floor Dysfunct. 2008;19(12):1603-1609.

36. Morgan DM, Rogers MA, Huebner M, Wei JT, Delancey JO. Heterogeneity in anatomic outcome of sacrospinous ligament fixation for prolapse: a systematic review. Obstet Gynecol. 2007;109(6): 1424-1433. 
37. Margulies RU, Rogers MA, Morgan DM. Outcomes of transvaginal uterosacral ligament suspension: systematic review and metaanalysis. Am J Obstet Gynecol. 2010;202(2):124-134.

38. Edenfield AL, Amundsen CL, Weidner AC, Wu JM, George A, Siddiqui NY. Vaginal prolapse recurrence after uterosacral ligament suspension in normal-weight compared with overweight and obese women. Obstet Gynecol. 2013;121(3):554-559.

39. Siddiqui NY, Mitchell TR, Bentley RC, Weidner AC. Neural entrapment during uterosacral ligament suspension: an anatomic study of female cadavers. Obstet Gynecol. 2010;116(3):708-713.

40. Schon Ybarra MA, Gutman RE, Rini D, Handa VL. Etiology of postuterosacral suspension neuropathies. Int Urogynecol J Pelvic Floor Dysfunct. 2009;20(9):1067-1071.

41. Flynn MK, Weidner AC, Amundsen CL. Sensory nerve injury after uterosacral ligament suspension. Am J Obstet Gynecol. 2006;195(6): 1869-1872.

42. Montoya TI, Luebbehusen HI, Schaffer JI, Wai CY, Rahn DD, Corton MM. Sensory neuropathy following suspension of the vaginal apex to the proximal uterosacral ligaments. Int Urogynecol J. 2012;23(12):1735-1740.

43. Barber MD, Brubaker L, Menefee S, et al. Operations and pelvic muscle training in the management of apical support loss (OPTIMAL) trial: design and methods. Contemp Clin Trials. 2009;30(2):178-189.

44. Nygaard IE, McCreery R, Brubaker L, et al. Abdominal sacrocolpopexy: a comprehensive review. Obstet Gynecol. 2004;104(4):805-823.

45. Nygaard I, Brubaker L, Zyczynski HM, et al. Long-term outcomes following abdominal sacrocolpopexy for pelvic organ prolapse. JAMA. 2013;309(19):2016-2024.

46. Culligan PJ, Salamon C, Priestley JL, Shariati A. Porcine dermis compared with polypropylene mesh for laparoscopic sacrocolpopexy: a randomized controlled trial. Obstet Gynecol. 2013;121(1):143-151.

47. Altman D, Anzen B, Brismar S, Lopez A, Zetterström J. Long-term outcome of abdominal sacrocolpopexy using xenograft compared with synthetic mesh. Urology. 2006;67(4):719-724.

48. Deprest J, De Ridder D, Roovers JP, Werbrouck E, Coremans G, Claerhout F. Medium term outcome of laparoscopic sacrocolpopexy with xenografts compared to synthetic grafts. J Urol. 2009;182(5):2362-2368

49. Culligan PJ, Blackwell L, Goldsmith LJ, Graham CA, Rogers A, Heit MH. A randomized controlled trial comparing fascia lata and synthetic mesh for sacral colpopexy. Obstet Gynecol. 2005;106(1):29-37.

50. Cundiff GW, Varner E, Visco AG, et al. Risk factors for mesh/ suture erosion following sacral colpopexy. Am J Obstet Gynecol. 2008;199(6):688. e1-e5.

51. Geller EJ, Siddiqui NY, Wu JM, Visco AG. Short-term outcomes of robotic sacrocolpopexy compared with abdominal sacrocolpopexy. Obstet Gynecol. 2008;112(6):1201-1206.

52. Hsiao KC, Latchamsetty K, Govier FE, Kozlowski P, Kobashi KC. Comparison of laparoscopic and abdominal sacrocolpopexy for the treatment of vaginal vault prolapse. J Endourol. 2007;21(8):926-930.

53. Khan A, Alperin M, Wu N, et al. Comparative outcomes of open versus laparoscopic sacrocolpopexy among Medicare beneficiaries. Int Urogynecol J. 2013;24(11):1883-1891.

54. Siddiqui NY, Geller EJ, Visco AG. Symptomatic and anatomic 1-year outcomes after robotic and abdominal sacrocolpopexy. Am J Obstet Gynecol. 2012;206(5):435.1-5.

55. Freeman RM, Pantazis K, Thomson A, et al. A randomised controlled trial of abdominal versus laparoscopic sacrocolpopexy for the treatment of post-hysterectomy vaginal vault prolapse: LAS study. Int Urogynecol J. 2013;24(3):377-384.
56. Ploumidis A, Spinoit AF, Naeyer GD, et al. Robot-assisted sacrocolpopexy for pelvic organ prolapse: surgical technique and outcomes at a single high-volume institution. Eur Urol. Epub June 11, 2013.

57. Geller EJ, Lin FC, Matthews CA. Analysis of robotic performance times to improve operative efficiency. J Minim Invasive Gynecol. 2013;20(1):43-48

58. Jonsson Funk M, Edenfield AL, Pate V, Visco AG, Weidner AC, Wu JM. Trends in use of surgical mesh for pelvic organ prolapse. Am J Obstet Gynecol. 2013;208(1):79. e1-e7.

59. US Food and Drug Administration. Update on serious complications associated with transvaginal placement of surgical mesh for pelvic organ prolapse. 2011. Available from: http://www.fda.gov/medicaldevices/ safety/alertsandnotices/ucm262435.htm. Accessed July 13, 2011.

60. US Food and Drug Administration. Urogynecologic surgical mesh: update on the safety and effectiveness of transvaginal placement for pelvic organ prolapse. 2011. Available from: http://www.fda.gov/ downloads/MedicalDevices/Safety/AlertsandNotices/UCM262760. pdf. Accessed October 31, 2013.

61. Abed H, Rahn DD, Lowenstein L, Balk EM, Clemons JL, Rogers RG. Incidence and management of graft erosion, wound granulation, and dyspareunia following vaginal prolapse repair with graft materials: a systematic review. Int Urogynecol J. 2011;22(7):789-798.

62. Diwadkar GB, Barber MD, Feiner B, Maher C, Jelovsek JE. Complication and reoperation rates after apical vaginal prolapse surgical repair: a systematic review. Obstet Gynecol. 2009;113(2 Pt 1):367-373.

63. Vu MK, Letko J, Jirschele K, et al. Minimal mesh repair for apical and anterior prolapse: initial anatomical and subjective outcomes. Int Urogynecol J. 2012;23(12):1753-1761.

64. NICHD Pelvic Floor Disorders Network. Study of uterine prolapse procedures - randomized trial (SUPeR). Available from: http:// clinicaltrials.gov/show/NCT01802281. NLM identifier: NCT01802281. Accessed October 31, 2013.

65. American Urogynecologic Society. Informed Consent Toolkit. 2012. Available from: http://www.augs.org/p/cm/ld/fid=174. Accessed October 31, 2013.

66. Brubaker L, Cundiff GW, Fine P, et al. Abdominal sacrocolpopexy with Burch colposuspension to reduce urinary stress incontinence. N Engl J Med. 2006;354(15):1557-1566.

67. Wei JT, Nygaard I, Richter HE, et al. A midurethral sling to reduce incontinence after vaginal prolapse repair. $N$ Engl J Med. 2012;366(25):2358-2367.

68. Ogah J, Cody DJ, Rogerson L. Minimally invasive synthetic suburethral sling operations for stress urinary incontinence in women: a short version Cochrane review. Neurourol Urodyn. 2011;30(3):284-291.

69. Jelovsek JE, Barber MD, Paraiso MF, Walters MD. Functional bowel and anorectal disorders in patients with pelvic organ prolapse and incontinence. Am J Obstet Gynecol. 2005;193(6):2105-2111.

70. Arya LA, Novi JM, Shaunik A, Morgan MA, Bradley CS. Pelvic organ prolapse, constipation, and dietary fiber intake in women: a case-control study. Am J Obstet Gynecol. 2005;192(5):1687-1691.

71. Saks EK, Harvie HS, Asfaw TS, Arya LA. Clinical significance of obstructive defecatory symptoms in women with pelvic organ prolapse. Int J Gynaecol Obstet. 2010;111(3):237-240.

72. Bradley CS, Nygaard IE, Brown MB, et al. Bowel symptoms in women 1 year after sacrocolpopexy. Am J Obstet Gynecol. 2007;197(6):642. e1-e8.

73. Gustilo-Ashby AM, Paraiso MF, Jelovsek JE, Walters MD, Barber MD. Bowel symptoms 1 year after surgery for prolapse: further analysis of a randomized trial of rectocele repair. Am J Obstet Gynecol. 2007;197(1):76. e1-e5. 
International Journal of Women's Health

Dovepress

\section{Publish your work in this journal}

The International Journal of Women's Health is an international, peerreviewed open-access journal publishing original research, reports, editorials, reviews and commentaries on all aspects of women's healthcare including gynecology, obstetrics, and breast cancer. The manuscript management system is completely online and includes Visit http://www.dovepress.com/testimonials.php to read real quotes from published authors.

Submit your manuscript here: http://www.dovepress.com/international-journal-of-womens-health-journal 